\title{
Does localization occur in a hierarchical random-matrix model for many-body states? ${ }^{1}$
}

\author{
Thomas Rupp ${ }^{\mathrm{a}, 2}$, Hans A. Weidenmüller ${ }^{\mathrm{a}}$, and Jean Richert ${ }^{\mathrm{b}}$ \\ ${ }^{a}$ Max-Planck-Institut für Kernphysik, Postfach 103980, 69029 Heidelberg, \\ Germany \\ ${ }^{\mathrm{b}}$ Laboratoire de Physique Théorique, Université Louis Pasteur, \\ 3 rue de l'Université, 67084 Strasbourg Cedex, France ${ }^{3}$
}

\begin{abstract}
We use random-matrix theory and supersymmetry techniques to work out the twopoint correlation function between states in a hierarchical model which employs Feshbach's chaining hypothesis: Classes of many-body states are introduced. Only states within the same or neighboring classes are coupled. We assume that the density of states per class grows monotonically with class index. The problem is mapped onto a one-dimensional non-linear sigma model. In the limit of a large number of states in each class we derive the critical exponent for the growth of the level density with class index for which delocalization sets in. From a realistic modelling of the class-dependence of the level density, we conclude that the model does not predict Fock-space localization in nuclei.
\end{abstract}

Key words: Fock-space localization, non-linear sigma model PACS: 24.10.Pa; 72.15.Rn

Statistical methods play an important role in the quantal many-body problem. We recall the importance of the Gaussian Orthogonal Ensemble of random matrices (the GOE) for the description of fluctuation properties of the energies and wave functions of neutron resonances and other data on nuclear energy levels located a few $\mathrm{MeV}$ above the ground state. The use of the GOE is not restricted to nuclei, of course, and this ensemble is equally important in other many-body systems like atoms or quantum dots [1].

It is well known that the GOE does not furnish a completely adequate description of stochasticity in any of these systems. This is because the forces

1 dedicated to Franz Wegner on the occasion of his 60th birthday

2 electronic mail: thomas.rupp@mpi-hd.mpg.de

3 Unité Mixte de Recherche CNRS - Université (UMR 7085) 
between the constituents (nucleons in the case of nuclei, electrons in the case of atoms and quantum dots) are predominantly of two-body type. Then it is more natural to assume that the matrix elements of the two-body interaction are random variables. Both in nuclear and in atomic physics, there is empirical evidence that this assumption is viable $[2,3]$. The random-matrix ensemble which implements this assumption in large shell-model spaces (the two-body random ensemble) differs from the GOE: The number of independent twobody matrix elements is generically much smaller than the dimension of a typical shell-model matrix, while for the GOE, the number of independent matrix elements is proportional to the square of the dimension of the matrix. Numerical studies have indicated long ago that this characteristic difference is immaterial for spectral fluctuation properties [4,5]. However, these studies were limited to small matrix dimensions. Therefore, the question persists whether the GOE and the random two-body ensemble are fully equivalent.

The question has resurfaced with the recent observation that in quantum dots, a statistical model for the two-body interaction allows, in principle, for localization in Fock space [6]. Numerical studies seem to support this proposal, at least at sufficiently high energies [7], while near the Fermi energy no evidence for localization was found [8]. It is then only natural to ask whether a random two-body interaction in nuclei might likewise lead to Fock-space localization in nuclei. Such localization would have exciting implications for level statistics and wave function properties. Moreover, Fock-space localization could lead to novel aspects of nuclear reaction dynamics.

Localization is a wave phenomenon discovered by Anderson [9]. It occurs when non-interacting electrons move diffusively under the influence of impurity scattering through a conductor. The Anderson tight-binding model describes this process in terms of a lattice with random on-site energies distributed uniformly over an interval of length $2 W$ and with fixed hopping matrix elements $V$ connecting neighboring sites. Localized eigenfunctions of the tight-binding Hamiltonian are not spread uniformly over the entire lattice but possess an envelope which decays exponentially at large distance. Anderson localization occurs not only in the passage of Schrödinger waves through a disordered medium but likewise in the passage of light through a medium with a disordered index of refraction [10].

The concept of localization can be extended to many-body systems. This was first suggested in quantum chemistry [11]. Logan and Wolynes observed a close topological similarity between a statistical description of the many-body problem in polyatomic molecules and the tight-binding model. An analogous similarity exists in quantum dots [6]. In the present paper, we point out that a corresponding similarity also exists in a Fock-space description of many-body systems like nuclei or atoms with random two-body interactions. We investigate the question whether in these systems, localization is expected to occur. 
The occurrence of localization can either be established by very extensive numerical calculations or analytically. Here, we choose the second approach. First, we must seek a model which allows us to pose quantitative questions. Optimally, we would want to consider a Hamiltonian which is the sum of onebody and two-body operators. The one-body terms would describe a nearly degenerate set of single-particle or shell-model orbitals, and the two-body terms would contain the random two-body matrix elements. This kind of model does not allow for an analytical treatment, however, and it is difficult to see how numerical calculations on the required scale could be performed. Our work is, instead, based on a Fock-space model commonly used in the statistical theory of nuclear reactions. We recall that in nucleon-induced nuclear reactions, precompound reactions are important whenever the equilibration time of the compound system is comparable to or even larger than the decay time of the system. This situation occurs typically at bombarding energies of several $10 \mathrm{MeV}$. Then, the standard compound-nucleus model (which uses the GOE) fails to apply and is replaced by a hierarchical statistical model: The incident nucleon creates a sequence of $m$-particle $(m-1)$-hole states where the integer $m$ increases by one unit in each two-body collision of the projectile with one of the target nucleons. The density of $m$-particle $(m-1)$-hole states at fixed excitation energy grows very strongly with increasing $m$, see Eq. (27) and the text following it. Therefore, a dynamical description of the process is out of the question and statistical concepts are used instead. We define classes of states. The states in class $m$ are composed of the $m$-particle $(m-1)$-hole states. Within each class, the two-body interaction mixes the $m$-particle $(m-1)$-hole states. We assume that the resulting matrix problem in each class $m$ can be replaced by a GOE. The GOE's pertaining to different classes (different $m$ values) are uncorrelated. For each $m$, the one parameter of the GOE is fixed by the density of states in that class. Classes differing in $m$ by one unit are connected by the elements of the two-body interaction which are taken to be independent Gaussian distributed random variables. This last assumption corresponds to Feshbach's "chaining hypothesis". It embodies the two-body character of the interaction and defines a hierarchical model. Within this model, we ask whether the eigenstates of the full Hamiltonian are localized. We now turn to the details of this model. We closely follow the work of Ref. [12].

We introduce a basis $|m \mu\rangle$ of states where $m$ is the class index introduced above, and where $\mu$ with $\mu=1, \ldots, N_{m}$ is a running index. The Hamiltonian reads

$$
H=\sum_{m n \mu \nu}|m \mu\rangle H_{m \mu, n \nu}\langle n \nu|
$$


and

$$
H_{m \mu, n \nu}=\delta_{m n} \delta_{\mu \nu} h_{m}+\delta H_{m \mu, n \nu}
$$

is real and symmetric under exchange of $(m \mu)$ and $(n \nu)$. We assume that all matrix elements are Gaussian distributed random variables with the following first and second moments,

$$
\begin{aligned}
\overline{H_{m \mu, n \nu}} & =\delta_{m n} \delta_{\mu \nu} h_{m}, \\
\overline{\delta H_{m \mu, n \nu} \delta H_{m^{\prime} \mu^{\prime}, n^{\prime} \nu^{\prime}}} & =M_{m n}\left(\delta_{m m^{\prime}} \delta_{n n^{\prime}} \delta_{\mu \mu^{\prime}} \delta_{\nu \nu^{\prime}}+\delta_{m n^{\prime}} \delta_{n m^{\prime}} \delta_{\mu \nu^{\prime}} \delta_{\nu \mu^{\prime}}\right) .
\end{aligned}
$$

The bar indicates ensemble averaging. For fixed $m$, the matrix $H_{m \mu, m \nu}$ belongs to the GOE. The matrices describing different classes $m$ are seen to be uncorrelated. In order to keep the GOE spectrum finite in the limit $N_{m} \rightarrow \infty$, the diagonal terms $M_{m m}$ must scale as $N_{m}^{-1}$. We consider the weak coupling case [12] where the non-diagonal elements $M_{m n}(m \neq n)$ are suppressed by an additional factor $N_{n}^{-1}$,

$$
M_{m n}=\frac{\lambda_{m}^{2}}{N_{m}} \delta_{m n}+\frac{\lambda_{m n}^{2}}{N_{m} N_{n}}\left(1-\delta_{m n}\right)
$$

Here $\lambda_{m}$ and $\lambda_{m n}$ are the strength of the matrix elements in class $m$ and the coupling strength of states in different classes, respectively. In keeping with the remarks made above, we assume that $\lambda_{m n}$ vanishes for $|m-n| \geq 2$. The spectrum of the $N_{m}$ states in class $m$ has the shape of a semicircle with center $h_{m}$ and radius $2 \lambda_{m}$. We proceed by assuming that all semicircles have identical centers, $h_{m}=0$ for all $m$, and identical radii, $\lambda_{m}=\lambda$ for all $m$. The remaining parameters $N_{m}$ allow us to account for the fact that the local level densities $\rho_{m}(E)$ at energy $E$ differ in the classes. We work at the centers of the semicircles and put $E=0$ without loss of generality. Later we need the inverse $g$ of $M$ given by

$$
g_{m n}=\frac{N_{m}}{\lambda^{2}} \delta_{m n}-\frac{\lambda_{m n}^{2}}{\lambda^{4}}\left(1-\delta_{m n}\right)+O\left(\frac{1}{\sqrt{N_{m} N_{n}}}\right) .
$$

With $D\left(E^{ \pm}\right)=E-H \pm i \eta$ and the ensemble-averaged two-point correlation function $\mathcal{K}$ defined by $(m \neq n)$

$$
\mathcal{K}_{m \mu, n \nu}=\overline{\left\langle m \mu\left|D^{-1}\left(E^{+}\right)\right| n \nu\right\rangle\left\langle n \nu\left|D^{-1}\left(E^{-}\right)\right| m \mu\right\rangle},
$$

the quantity of central interest is the class average of $\mathcal{K}$,

$$
\mathcal{K}_{m n}=\frac{1}{N_{m} N_{n}} \sum_{\mu \nu} \mathcal{K}_{m \mu, n \nu}
$$


If $\mathcal{K}_{m n}$ vanishes exponentially with increasing distance $|m-n|$, the eigenstates of $H$ are (exponentially) localized.

The explicit dependence of $\mathcal{K}_{m n}$ on $|m-n|$ can be found with the help of the supersymmetric non-linear sigma model [13-15] the use of which is by now completely standard. Moreover, a derivation taylored to the present problem may be found in Ref. [12]. Therefore, we restrict ourselves to the essential steps and emphasize those aspects which are specific to the present problem. We use the notation of Ref. [15]. The correlation function is expressed in terms of the generating function $Z$,

$$
\mathcal{K}_{m n}=\left.\frac{\partial^{2} \bar{Z}(J)}{\partial J_{m}^{15} \partial J_{n}^{15}}\right|_{J=0}
$$

where $J_{m}^{15}$ denotes the $(1,5)$ element (with respect to the supersymmetry indices) of the auxiliary $J$-field. Similar notation is used in Eq. (15). Here we have averaged the generating function over the Gaussian distribution of $H_{m \mu, n \nu}$. After eliminating the quartic dependence on the original integration variables by means of a Hubbard-Stratonovitch transformation, we find for $\bar{Z}$ the expression

$$
\begin{aligned}
& \bar{Z}(E, J)= \\
& \int d[\sigma] \exp \left(-\frac{1}{4} \sum_{m n} \lambda^{2} g_{m n} \operatorname{trg}\left(\sigma_{m} \sigma_{n}\right)-\frac{1}{2} \operatorname{trg}_{m, \mu, \alpha} \ln \mathcal{N}(J)\right) .
\end{aligned}
$$

The fields $\sigma_{m}$ are $8 \times 8$ graded matrices and $d[\sigma]=\prod_{m} d\left[\sigma_{m}\right]$. Moreover,

$$
\mathcal{N}(J)=E+i \eta+J-\Sigma, \quad \Sigma=\left\{\lambda \delta_{m n} \delta_{\mu \nu} \sigma_{m}^{\alpha \beta}\right\}
$$

The integral in Eq. (10) is evaluated by means of a saddle-point approximation by varying the $\sigma_{m}$ 's. In view of the smallness of the terms with $m \neq n$ in Eq. (6), we obtain a separate saddle-point equation $\sigma_{m}\left(E-\lambda \sigma_{m}\right)=\lambda$ for each class $m$. The solution yields the semicircle for the level density. Integrating over the massive modes in the limit where $N_{m} \rightarrow \infty$ leaves us only the Goldstone modes $\sigma_{m}^{\mathrm{G}}$. We focus attention on the correlation function $\mathcal{K}(m)=\mathcal{K}_{1 m}$ which takes the form

$$
\mathcal{K}(m)=\frac{1}{2 \lambda^{2}} \int \prod_{k=1}^{M} d\left[\sigma_{k}\right]\left(\sigma_{1}^{\mathrm{G}}\right)^{51}\left(\sigma_{m}^{\mathrm{G}}\right)^{51} \exp \left(\sum_{i=1}^{M-1} \frac{\lambda_{i i+1}^{2}}{4 \lambda^{2}} \operatorname{trg}\left(\sigma_{i}^{\mathrm{G}} \sigma_{i+1}^{\mathrm{G}}\right)\right) .
$$

Here $M$ is the total number of classes. In the case of weak coupling we have [12]

$$
4 \frac{\lambda_{m n}^{2}}{\lambda^{2}}=2 \pi \rho_{m} \overline{V_{m n}^{2}} 2 \pi \rho_{n}
$$


where we wrote $M_{m n}=\overline{V_{m n}{ }^{2}}$ and where $\rho_{m}=N_{m} / \pi \lambda$ is the density of states in class $m$ at energy $E=0$. The form of the expression in the exponent of Eq. (12) displays the chaining hypothesis (only states belonging to nextneighboring classes are coupled by non-vanishing matrix elements).

To proceed, we omit from now on the index $G$ on the sigma fields and follow Refs. $[16,17]$. The aim consists in replacing the summation over classes by an integration (continuum limit). We put

$$
\frac{\lambda_{i i+1}^{2}}{4 \lambda^{2}}=\frac{1}{4 \epsilon} \alpha_{i, i+1}
$$

with $\epsilon=1 / \zeta$ and both $\zeta$ and $\alpha_{i, i+1} \gg 1$. This is justified because the left-hand side of Eq. (14) is much larger than unity, see Eqs. (26) and (27). We write the correlation function in the form

$$
\mathcal{K}(m)=\frac{1}{2 \lambda^{2}} \int d \sigma_{1} d \sigma_{m} \sigma_{1}^{51} W\left(\sigma_{1}, \sigma_{m} ; 1, m\right) \sigma_{m}^{51} Y\left(\sigma_{m} ; m\right)
$$

where

$$
\begin{aligned}
W\left(\sigma_{m}, \sigma_{n} ; m, n\right) & =\int \prod_{k=m+1}^{n-1} d \sigma_{k} \exp \left[\frac{1}{4 \epsilon} \sum_{i=m}^{n-1} \alpha_{i, i+1} \operatorname{trg}\left(\sigma_{i} \sigma_{i+1}\right)\right] \\
Y\left(\sigma_{m} ; m\right) & =\int \prod_{k=m+1}^{M} d \sigma_{k} \exp \left[\frac{1}{4 \epsilon} \sum_{i=m}^{M-1} \alpha_{i, i+1} \operatorname{trg}\left(\sigma_{i} \sigma_{i+1}\right)\right] .
\end{aligned}
$$

Obviously, $W$ obeys the equation

$$
\begin{aligned}
& W\left(\sigma_{m}, \sigma_{n+1} ; m, n+1\right) \\
& =\int d \sigma_{n} W\left(\sigma_{m}, \sigma_{n} ; m, n\right) \exp \left[\frac{1}{4 \epsilon} \alpha_{n, n+1} \operatorname{trg}\left(\sigma_{n} \sigma_{n+1}\right)\right] .
\end{aligned}
$$

We use the smallness of $\epsilon$ to take the continuum limit and replace the summation over classes by an integration over the continuous variable $t=m / \zeta$. Then,

$$
\begin{aligned}
\alpha_{m, m+1} & \rightarrow \alpha(t) \\
W\left(\sigma_{1}, \sigma_{m} ; 1, m\right) & \rightarrow W\left(\sigma_{1}, \sigma(t) ; t\right) \\
Y\left(\sigma_{m} ; m\right) & \rightarrow Y(\sigma(t) ; t)
\end{aligned}
$$

and Eq. (17) becomes an integral equation. This integral equation is equivalent 
to a modified heat equation,

$$
\alpha(t) \partial_{t} W\left(\sigma^{\prime}, \sigma ; t\right)=\Delta_{\sigma} W\left(\sigma^{\prime}, \sigma ; t\right)
$$

with the initial condition

$$
\lim _{t \rightarrow 0} W\left(\sigma^{\prime}, \sigma ; t\right)=\delta\left(\sigma^{\prime}-\sigma\right)
$$

Here $\Delta_{\sigma} \equiv \partial^{2} / \partial \sigma^{2}[16,17]$

In previous work $[13,17]$, the prefactor in the exponent of Eq. (12) was a constant (independent of the class index $i$ ), and the solution of the heat equation did display an exponential decay signalling localization. In the present case, $\alpha(t)$ does depend upon $t$ because the level densities $\rho_{m}$ depend strongly upon $m$. This fact raises the following questions: (i) Is localization affected or even destroyed by a monotonic increase of $\rho_{m}$ with $m$ and, thus, of $\alpha(t)$ with $t$ ? (ii) If so, can we determine the critical exponent $\beta$ in $\alpha(t) \propto t^{\beta}$ which marks the transition from the localized to the delocalized regime? (iii) What are the implications of our findings for localization in nuclei, i.e., for a realistic modelling of the $t$-dependence of $\alpha(t)$ ?

To answer these questions, we observe that we retrieve the standard heat equation by a change of variable,

$$
\alpha(t) \partial_{t}=\partial_{s}
$$

In terms of the rescaled function

$$
\tilde{W}\left(\sigma_{1}, \tilde{\sigma}(s) ; s\right)=\tilde{W}\left(\sigma_{1}, \tilde{\sigma}(s(t)) ; s(t)\right)=W\left(\sigma_{1}, \sigma(t) ; t\right)
$$

this leads to

$$
\partial_{s} \tilde{W}\left(\tilde{\sigma}^{\prime}, \tilde{\sigma} ; s\right)=\Delta_{\tilde{\sigma}} \tilde{W}\left(\tilde{\sigma}^{\prime}, \tilde{\sigma} ; s\right)
$$

We choose $s(t)$ such that $s(0)=0$ which ensures that $\tilde{W}$ also obeys Eq. (19). The transformation (20) reduces our problem to the heat equation solved in Ref. [13]. The rescaled propagator

$$
\tilde{\mathcal{K}}(s)=\frac{1}{2 \lambda^{2}} \int d \sigma_{1} d \tilde{\sigma}(s) \sigma_{1}^{51} \tilde{W}\left(\sigma_{1}, \tilde{\sigma}(s) ; s\right) \tilde{\sigma}^{51}(s) \tilde{Y}(\sigma(s) ; s)
$$

can be worked out by introducing the Fourier transform $\tilde{K}(k)$ of Eq. (23). 
This yields

$$
\tilde{\mathcal{K}}(s)=\frac{\pi^{4} \sqrt{\pi}}{16 \zeta \lambda^{2}}\left(\frac{4 \zeta}{s}\right)^{3 / 2} \exp \left(\frac{-s}{4 \zeta}\right)
$$

which is valid for $s \gg \zeta$. The function $\mathcal{K}(t)$ is obtained by solving for $s(t)$ the differential equation

$$
\frac{d s(t)}{d t}=\frac{1}{\alpha(t)}
$$

and replacing $s$ by $s(t)$ in Eq. (24). For a power-law dependence, $\alpha(t) \propto t^{\beta}$, Eq. (8) yields $s \propto t^{1-\beta}$. Combining this with Eq. (24), we see that $\beta=1$ is the critical exponent which marks the transition from localization to delocalization. The existence of such an exponent is intuitively clear: A monotonic increase of $\alpha(t)$ with $t$ signals an ever increasing phase space. The resulting drag on the system overcompensates, for $\beta>1$, the tendency of the system to form localized states.

In order to apply this result to the case of nuclei, we must determine the dependence of $\alpha(t)$ on $t$. We recall that

$$
\alpha(t) \Leftrightarrow \alpha_{m, m+1}=\left(\pi^{2} \rho_{m} \overline{V_{m, m+1}^{2}} \rho_{m+1}\right) / \zeta=\left(\pi^{2} \rho_{m} \Gamma_{m \rightarrow m+1}^{\downarrow}\right) / \zeta
$$

where $\Gamma_{m \rightarrow m+1}^{\downarrow}$ is the spreading width for transitions from class $m$ to class $m+1$. The width $\Gamma_{m \rightarrow m+1}^{\downarrow}$ approximately scales with $1 / m$ [18]. The density of states in class $m$ at excitation energy $E$ is [19]

$$
\rho_{m}(E)=\frac{1}{m !(m+1) !(2 m) ! \Delta}\left(\frac{E}{\Delta}\right)^{2 m}
$$

where $\Delta$ is the mean level spacing of the single-particle states. For fixed $E$, $\rho_{m}(E)$ grows strongly with $m$ up to a maximal value of $m$ and then drops quickly. In the present context, we are not interested in this cut-off because it might mask the existence of localization. For this reason, we take the limit of high excitation energy $E / \Delta \gg m$. Then, we can neglect the factorial factors in Eq. (27) as well as the $1 / m$ dependence of $\Gamma_{m \rightarrow m+1}$ and obtain approximately $\alpha_{m, m+1} \propto(E / \Delta)^{2 m}$. This yields

$$
s(t) \Leftrightarrow s_{m} \propto 1-(E / \Delta)^{-2 m} .
$$

We observe that $\alpha_{m, m+1}$ grows with $m$ much more strongly than linearly. As a consequence, $s_{m}$ is bounded from above and approaches asymptotically a 
finite constant. Thus, the correlation function $\mathcal{K}(t)$ tends to a finite value as $t \rightarrow \infty$. We conclude that the interaction spreads the states in class 1 over the entire Hilbert space.

The results of the model investigated in the present paper negate the possibility of localization in a many-body Fermi system like the nucleus. This finding must be contrasted with the results of Ref. [6] where the chain of $m$-particle $(m-1)$-hole states was mapped onto a Bethe lattice, and localization was predicted. Such mapping completely neglects the interaction between states within each class $m$. In contradistinction, our use of a GOE for the states within each class may overemphasize the role of the interaction between states within each class $m$, although we believe the present model to be closer to reality in nuclei than the model using a Bethe lattice. As mentioned above, in the numerical work of Leyronas et al. [7] evidence for localization was presented. In that work, all those $m$-particle $(m-1)$-hole states were taken into

account the unperturbed energies of which lie in some small neighborhood of the energy $E$ considered. It is not clear to us whether this constraint might not affect the localization properties of the system.

In summary, we have shown that in the framework of our hierarchical model localization is destroyed whenever the density of states increases more strongly than linearly with increasing complexity $m$ of states. In the case of nuclei, the model does not predict localization.

T.R. thanks C. Mejía-Monasterio for beneficial discussions at the early stages of this work.

\section{References}

[1] T. Guhr, A. Müller-Groeling, and H.A. Weidenmüller, Phys. Rep. 299 (1998) 189.

[2] A. Gervois, Phys. Lett. B 26 (1968) 413.

[3] V.V. Flambaum, A.A. Gribakina, G.F. Gribakin, and M.G. Kozlov, Phys. Rev. A 50 (1994) 267.

[4] J.B. French and S.S.M. Wong, Phys. Lett. B 35 (1971) 5.

[5] O. Bohigas and J. Flores, Phys. Lett. B 35 (1971) 383.

[6] B.L. Altshuler, Y. Gefen, A. Kamenev, and L.S. Levitov, Phys. Rev. Lett. 78 (1997) 2803.

[7] X. Leyronas, J. Tworzydło, and C.W.J. Beenakker, Phys. Rev. Lett. 82 (1999) 4894. 
[8] C. Mejía-Monasterio, J. Richert, T. Rupp, and H.A. Weidenmüller, Phys. Rev. Lett. 81 (1998) 5189.

[9] P.W. Anderson, Phys. Rev. 109 (1958) 1492.

[10] D.S. Wiersma, P. Bartolini, A. Lagendijk, and R. Righini, Nature 390 (1997) 671.

[11] D.E. Logan and P.G. Wolynes, J. Chem. Phys. 93 (1990) 4994.

[12] H. Nishioka, J.J.M. Verbaarschot, H.A. Weidenmüller, and S. Yoshida, Ann. Phys. (N.Y.) 172 (1986) 67.

[13] K.B. Efetov, Adv. Phys. 32 (1983) 53.

[14] K.B. Efetov, Supersymmetry in disorder and chaos (Cambridge University Press, Cambridge, 1997).

[15] J.J.M. Verbaarschot, H.A. Weidenmüller, and M.R. Zirnbauer, Phys. Rep. 129 (1985) 367.

[16] M.R. Zirnbauer, Phys. Rev. Lett. 69 (1992) 1584.

[17] A.D. Mirlin, A. Müller-Groeling, and M.R. Zirnbauer, Ann. Phys. (N.Y.) 236 (1994) 325.

[18] M. Herman, G. Reffo, and H.A. Weidenmüller, Nucl. Phys. A 536 (1992) 124.

[19] A.D. Mirlin and Y.V. Fyodorov, Phys. Rev. B 56 (1997) 13393. 